\title{
Optimization using Nimrod/O and its Application to Robust Mechanical Design
}

\author{
Tom Peachey ${ }^{1}$, David Abramson ${ }^{1}$, Andrew Lewis ${ }^{2}$, Donny Kurniawan ${ }^{1}$ and Rhys \\ Jones $^{3}$ \\ ${ }^{1}$ School of Computer Science and Software Engineering, Monash University, Melbourne, \\ Australia \\ ${ }^{2}$ Division of Information Services, Griffith University, Brisbane, Australia \\ ${ }^{3}$ Department of Mechanical Engineering, Monash University, Melbourne, Australia
}

\begin{abstract}
We describe the Nimrod/O distributed optimization tool and its application to a problem in mechanical design. The problem is to determine the shape for a hole in a thin plate under load that gives optimal life in the presence of flaws. The experiment reveals two distinct design strategies for optimizing this life. Nimrod/O is able to find both of these rapidly due to its inherent parallelism.
\end{abstract}

\section{$1 \mathrm{Nimrod} / \mathrm{O}$}

Advances in computing science and engineering modeling have enabled design engineers to use computational models instead of real world prototypes in many situations. Such an approach is usually faster and cheaper and hence allows the user to explore various design scenarios. In particular the user may search through combinations of design parameters in order to achieve a design that is optimal in some sense. Similarly, scientific research is increasingly using computational models. Often the aim is to determine those model parameters that produce the best fit to realworld data so again the model is used repeatedly to achieve some optimum.

Such models typically require computational times of minutes or hours, even on a high end processor. We have produced a number of tools that facilitate concurrent execution of these models. The tools Nimrod and EnFuzion [1] allow the user to specify a range of values for each design parameter; then all combinations of these values are generated and the resulting jobs are farmed to a cluster of processors. Nimrod/G [2] is a version of Nimrod that utilises resources on a global computational grid. These tools may be used for design optimization by exploring all possibilities in a search space. A more efficient search is provided by Nimrod/O [3],[4],[5] which offers a variety of standard optimization procedures to explore the parameter space.

When a procedure requires values of the objective function it passes the parameters to Nimrod or EnFuzion for evaluation against the computational model. The model is run on an appropriate platform and the objective function value is extracted from the model output. A cache is superimposed between Nimrod/O and the backend to reduce the number of calculations required if the same parameter values are requested more 
then once. A persistent database is attached to the cache to support restart if Nimrod/O is terminated prematurely. The Nimrod/O implementation of the optimization procedures uses concurrent evaluations where possible. In addition it allows separate optimizations to be run concurrently.

To perform a Nimrod/O optimization the user needs to prepare a simple text "schedule" file. This specifies the parameters of the search space, any constraints imposed on the solution, how to run the computational model to obtain objective values and the optimization method(s) to apply. Assuming the model correctly executes on the remote nodes, no further development is required.

\section{Computation of Fatigue Life for Shape Optimization}

In this paper we discuss the application of Nimrod/O to the optimal design of mechanical components. The failure of such components is usually due to slow growth of a pre-existing crack followed by a sudden fracture [6]. A common mechanism for crack growth is fatigue due to cyclical loading. As a load is applied the high stress at the crack tip causes plastic deformation which produces an irreversible growth in the crack. This growth is small (typically $10^{-7}-10^{-3} \mathrm{~mm}$ ) but repeated cycles of loading may extend the crack to a stage where fracture occurs. The problem is very important industrially, because failures of large structures like trains, automobiles, oil drilling rigs and mining equipment can cost millions of dollars, and cause death and injury to personnel.

The computational model that we used is described in [7]. In this approach, finite element techniques are used to compute the stress field throughout the component for a given applied load in the absence of cracks. Then cracks are assumed to occur at critical boundaries of the component and a recent modification [8] of the finite element alternating method [9] is applied to compute the stress intensity factor at the crack positions. Finally the Paris growth law [10] is used to compute the number of loading cycles required for the cracks to grow from its given initial size to a given final size. The number of cycles at the worst (least cycles) crack is taken as the fatigue life. Because a fine numerical mesh is required at the crack tips, computation times are typically of the order of minutes even for small two-dimensional models.

For the purposes of the stress analysis and subsequent fracture mechanics the shape profile is defined by control points. The finite element analysis proceeds by fitting a spline through the points to generate further boundary points. For optimization purposes the control points may be considered the state variables in the search space. For example if a curve in two-dimensions is specified by 50 movable points then the optimization has 100 independent variables. Optimization in a space of such high dimension is notoriously difficult. A further difficulty is how to impose desired constraints; especially difficult are constraints on the slope of the boundary.

Despite these difficulties some success has been achieved using a "biological algorithm" which moves each control point normal to the boundary a distance based on the assessment of that point. Earlier work assessed points on the basis of stress [11] or stress intensity factor [12],[13],[14]; more recently [7] fatigue life has been used. 
An alternative approach, which is proposed in this paper, is to use a family of curves or surfaces specified by some parameters and to generate the control points from the curve. Finding the optimum shape involves determining the best parameters. Since the number of parameters is small, optimization is more efficient. However for this approach to find the true optimum the family of curves should include that optimum. Construction of a suitable family of curves can be a difficult design problem.

\section{The Test Problem}

The test problem is an aluminium alloy plate of thickness $1.5 \mathrm{~mm}$ with dimensions $320 \mathrm{~mm}$ by $600 \mathrm{~mm}$ as shown in Figure 1 . A remote stress field of $100 \mathrm{MPa}$ is applied in the vertical direction. The plate contains a hole of width $20 \mathrm{~mm}$ which is $10 \mathrm{~mm}$ from the left edge. As the geometry is symmetrical about the horizontal mid plane only the top half is modeled. This simple test problem is characteristic of a number of real designs found in practice. For example, the plate between the rear seats and the trunk of a passenger car, typically has a section removed to allow long items to project from the trunk into the cabin. In general, the hole needs to be as large as possible without compromising the mechanical stability of the plate, which is an important part of the structure of the car. In another setting, the same basic design can be found in the fuel tanks of high performance jet fighters. In this case a mechanical stiffener is placed in the fuel tank, which is in turn held in the wing, to increase the stability of the wing structure. However, a hole must be placed in the stiffener to allow fuel to move freely. Again, the hole needs to be large enough to allow fuel to move, but not too large that the effect of the stiffener is compromised.

The aim of the experiment is to determine the hole shape for optimal fatigue life in the presence of cracks distributed around the hole profile. The cracks have an initial length of $1 \mathrm{~mm}$ and are deemed to have failed when they reach a length of $5 \mathrm{~mm}$.

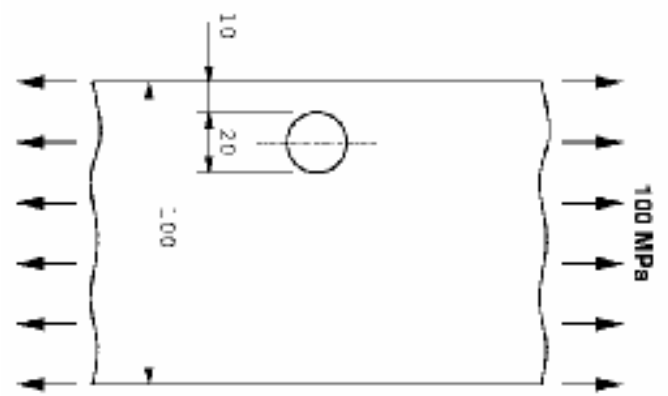

Fig. 1. Test problem for hole profile optimization. 


\section{Parameterization}

Theory predicts that optimal hole profile must be free of sharp corners and symmetric about the horizontal mid plane. A suitable family of smooth curves, symmetrical about the point $(p, q)$, are the "generalized ellipses",

$$
\frac{|x-p|^{t}}{a^{t}}+\frac{|y-q|^{t}}{b^{t}}=1 \text {. }
$$

These are closed curves of width $2 a$ height $2 b$ where $t$ controls the curvature at the shoulders of the curves. We will call this the "tightness". Figure 2 a shows some of these with $a=10, b=20$ and a variety of values of $t$. More generally, the curves

$$
\frac{|x-p|^{t}}{a^{t}}+\frac{|y-q|^{t} e^{-i \beta(x-p)}}{b^{t}}=1
$$

also have width $2 a$ and height $2 b$ at the vertical axis. The inclusion of the exponential factor allows for asymmetry about that axis. The parameter $\beta$, called the "bias" here, controls the slope at the top. Figure $2 \mathrm{~b}$ shows samples with $p=0, q=0, a=10$, $b=20, t=5$ and various $\beta$.

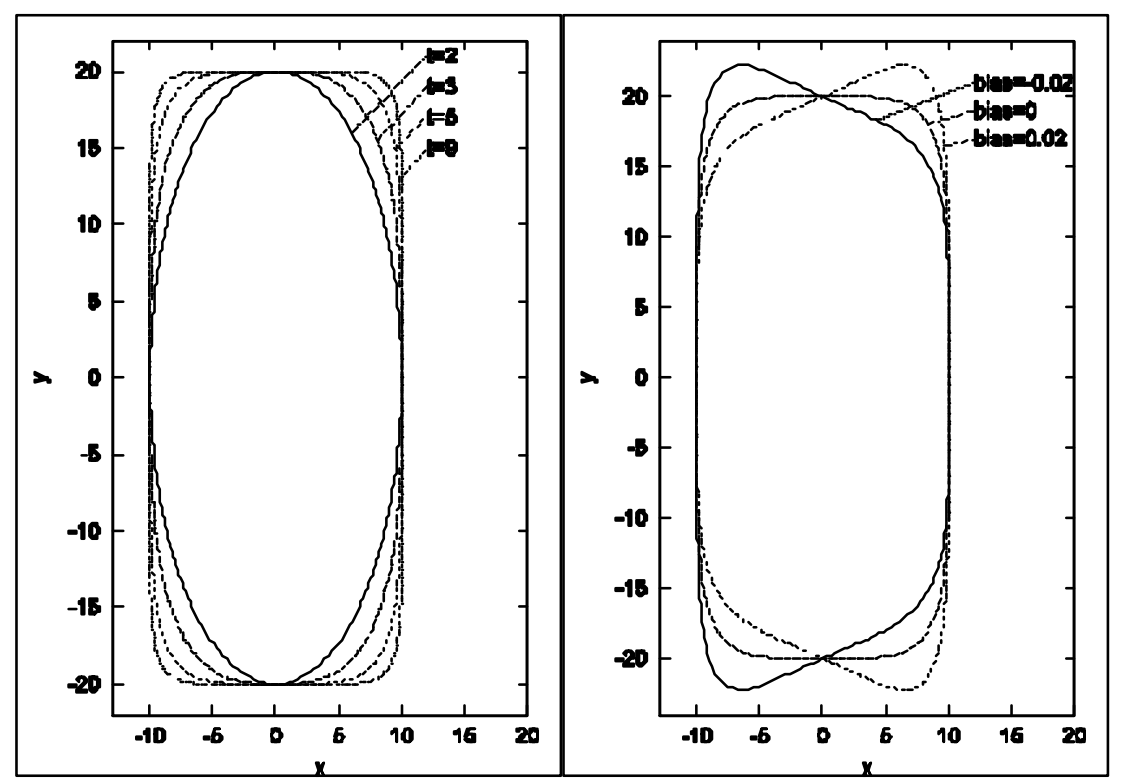

Fig. 2. Parametrized families (a) Equation (1), (b) Equation (2).

For the plate model we employed $a=10, p=20$, and $q=0$ giving a hole of width $20 \mathrm{~mm}$ which is $10 \mathrm{~mm}$ from the left boundary. Thus the variables $b, t$ and $\beta$ 
are the optimization parameters. The search space used was the domain $5 \leq b \leq 35$, $2 \leq t \leq 9,-0.02 \leq \beta \leq 0.02$.

\section{Results}

\section{Experiment 1}

Experiments were performed on a Compaq SC cluster, consisting of 20 Alpha EV68 processors connected by a Quadrics switch. Nine independent optimizations were performed starting from various initial parameters evenly spread through the search space. Each optimization used the simplex search algorithm [15]. The results are shown in Table 1.

The "parameters" referred are the triples $(b, t, \beta)$. As well as the final parameters and the optimum achieved, Nimrod/O reports statistics on the search process. The "Itrs" column gives the number of iterations of the simplex search. The "Evals" column contains two numbers; the second (in parentheses) is the number of evaluations required by the algorithm. However some evaluations may be duplicates, in which case the result is obtained from a cache rather than re-computed. The first number gives the number of unique evaluations. Since jobs are computed concurrently in batches, the number of batches gives a better indication of the running time of an optimization. The "Batches" column gives the number of batches requested by the algorithm and the number actually calculated. Because all nine optimizations were performed in parallel and batches averaged about 4 jobs, the concurrency was approximately 36 when compared with a serial optimizer performing the same searches.

\begin{tabular}{|c|c|c|c|c|c|}
\hline $\begin{array}{c}\text { Starting } \\
\text { Parameters }\end{array}$ & $\begin{array}{l}\text { Optimum } \\
\text { Parameters }\end{array}$ & $\begin{array}{l}\text { Optim. } \\
\text { Life }\end{array}$ & Itrs. & Evals. & Batches \\
\hline$(10,2,0)$ & $(8.72,2.000,-0.00032)$ & 3719 & 23 & $88(104)$ & $26(26)$ \\
\hline$(10,5.5,0)$ & $(35.00,4.390,-0.01687)$ & 5335 & 19 & $76 \quad(76)$ & $19(19)$ \\
\hline$(10,9,0)$ & $(35.00,8.986,-0.00104)$ & 5341 & 17 & $65 \quad(68)$ & $17(17)$ \\
\hline$(20,2,0)$ & $(33.86,4.078,-0.01904)$ & 5361 & 18 & $70 \quad(72)$ & $18(18)$ \\
\hline$(20,5.5,0)$ & $(33.89,4.523,-0.01569)$ & 5282 & 11 & $44 \quad(44)$ & $11(11)$ \\
\hline$(20,9,0)$ & $(35.00,7.963,0.00148)$ & 5316 & 12 & $48 \quad(48)$ & $12(12)$ \\
\hline$(30,2,0)$ & $(35.00,8.780,0.00047)$ & 5342 & 16 & $62 \quad(64)$ & $16(16)$ \\
\hline$(30,5.5,0)$ & $(35.00,8.300,0.00000)$ & 5342 & 27 & $31 \quad(32)$ & $8 \quad(8)$ \\
\hline$(30,9,0)$ & $(35.00,8.767,0.00267)$ & 5339 & 8 & $20 \quad(20)$ & $5 \quad(5)$ \\
\hline
\end{tabular}

Table 1. Results of 9 simplex searches..

Eight of the searches achieved an optimum with $b$ at or close to the upper limit of 35. This agrees with previous work [13] on this problem using a control point 
algorithm. that found the height of the hole grows to its upper bound. However one search revealed a local optimum in another part of the search space.

\section{Experiment 2}

So as to obtain a better understanding of the search space we used Nimrod to survey the search space evaluating the life at all combinations of $b=5,35(5), t=2,9(0.5)$, $\beta=-0.020,0.020(0.002)$. Maximum stress on the hole profile was also computed in each case.

For each of these values of the bias $\beta$, rubber sheet representations were produced for both the life and the stress as functions of $b$ and $t$. Further, isosurface representations were produced for life and stress in terms of the three variable $(b, t, \beta)$. These pictures are available at [16]. They show that the bias has only a minor effect on stress and life. For the case $\beta=0$, the rubber sheets for life and stress are shown in Figures $3 \mathrm{a}$ and $\mathrm{b}$ respectively.

Figure $3 \mathrm{a}$ shows two regions of the search space that give high values for life. One is around $b=35.0, t=9.0$ (we call this region 1) and the other near $b=5.0$,

$t=2.0$ (region 2). This explains why Nimrod/O searches found two distinct local optima. The fact that these optimal regions are on the search space boundaries suggest that extending the boundaries will allow better optima.

We expect that shapes with long life will have low stress. Figure $3 \mathrm{~b}$ shows that this is true for region 1 but not for region 2. The latter case seems anomalous in that maximum life corresponds to maximum stress. This matter is resolved by visualizations of the stress field for shapes taken from these regions. Figure 4a shows the hole shape and associated stress field for the shape $b=35.0 t=4.00$ and $\beta=-0.020$, which gave the global optimum in region 1 , and Figure $4 \mathrm{~b}$ for shape $b=5.0 t=2.00$ and $\beta=-0.004$, the local optimum in region 2 . The latter reveals that, although the shape gives high stress on the hole boundary, the stress falls off rapidly when moving into the body. Hence cracks on this boundary will initially grow quickly but this is balanced by very slow subsequent growth.

\section{Conclusion}

For the test geometry both long angular shapes and short rounded shapes produced high fatigue life, but only the former corresponds to low stress on the hole boundary. These results confirm that optimization for fatigue life may produce very different results from traditional optimization based on boundary stress. However, the current practice in industry is to only design for stress minimization. As a result of this work it would appear that to achieve this goal it may be best to use a fracture/fatigue rather than a stress based optimization process. This has clear implications for both safety and economics of production.

For this problem the search space contained multiple local optima; this fact was revealed by performing multiple searches from various starting points. Nimrod/O 
facilitated this by performing these searches in parallel. To understand these results a full survey of the search space was required. Here Nimrod was an appropriate tool.

\section{References}

1. Abramson D., Sosic R., Giddy J. and Hall B., "Nimrod: A Tool for Performing Parametised Simulations using Distributed Workstations", The 4th IEEE Symposium on High Performance Distributed Computing, Virginia, August 1995.

2. Buyya R, Abramson D., Giddy J.. "Nimrod/G: an architecture for a resource Management and Scheduling system in a Global Computational Grid", 4th International Conference on High-Performance computing in the Asia-Pacific Region (HPC Asia), Beijing, May 2000, 283-289.

3. Abramson D., Lewis, A. and Peachey, T., "Nimrod/O: A Tool for Automatic Design Optimization", The 4th International Conference on Algorithms \& Architectures for Parallel Processing (ICA3PP 2000), Hong Kong, December 2000.

4. Abramson D., Lewis, A. and Peachey, T. "Case Studies in Automatic Design Optimisation using the P-BFGS Algorithm”, 2001 High Performance Computing Symposium (HPC'01), Advanced Simulation Technologies Conference, Seattle, Washington (USA), April 2001, 104-109.

5. Abramson D, Lewis A, Peachey T, Fletcher, C., "An Automatic Design Optimization Tool and its Application to Computational Fluid Dynamics", SuperComputing 2001, Denver, Nov 2001.

6. Broek D., The practical use of fracture mechanics, Kluwer Academic Publishers, ISBN 0-7923-0223-0, 1988.

7. Jones R., Peng D., Chaperon P., Tan M., Abramsos D. and Peachey T. "Structural Optimization with Damage Tolerance Constraints", J. Theoretical and Applied Fracture Mechanics, (in press).

8. R. Jones and D. Peng, "A simple method for computing the stress intensity factors for cracks at notches", Engng. Failure Analysis, 9 (2002) 683-702.

9. Nishioka, T. and Atluri, S.N., "Analytical Solution for Embedded Elliptical Cracks, and Finite Element Alternating Method for Elliptical Surface Cracks, Subject to Arbitrary Loadings", Engng. Fracture Mechanics, Vol. 17, No. 3, (1983), 247-268.

10.Paris P. C. and Erdogan F. "A critical analysis of crack propogation laws", Trans. ASME, J. Basic Engng., Vol. 85, (1963), 528-534.

11.C. Mattheck and S. Burkhardt, "A new method of structural shape optimization based on biological growth", Int. J. Fatigue, Vol.12, No.3, (1990), 185-190.

12.P. Chaperon and R. Jones, M. Heller, S. Pitt and F. Rose, A methodology for structural optimisation with damage tolerance constraints, Journal of Engng. Failure Analysis, 7, (2000), 281-300.

13.R. Jones, P. Chaperon, and J. P. G. Sawyer, "Structural optimisation with damage tolerance constraints", Ageing Studies Lifetime Extension of Materials, , Edited by L. G. Mallinson, Kluwer Academic Press/Plenum Publishers, New York, ISBN 0306-46477-2, (2001), 601-608. 
14.R. Jones, P. Chaperon and M. Heller, "Structural optimisation with fracture strength constraints", J. of Engng. Fracture Mechanics, 69, (2002), 45-67.

15.Nelder, J. A. and Mead R., "A simplex method for function minimization", The Computer Journal, 7, (1965) 308-313.

16. http://www.csse.monash.edu.au/ donny/mc2/tcp/paper/
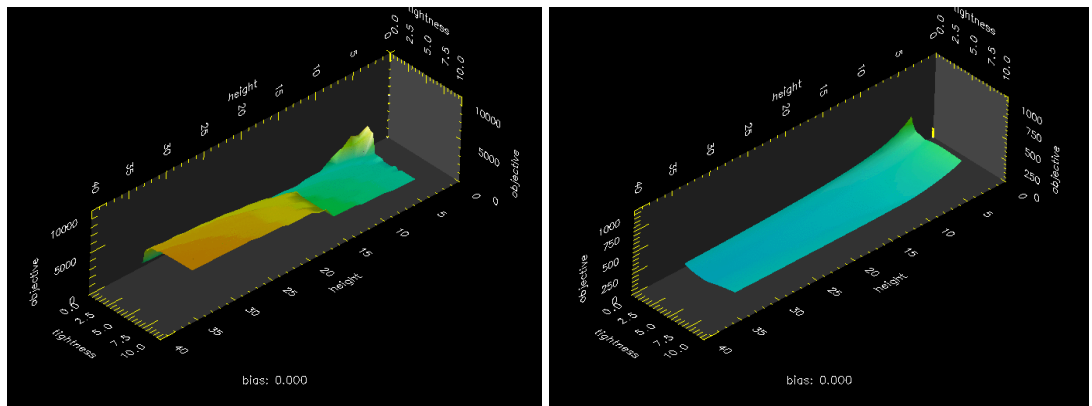

Fig. 3. Rubber sheet representations for (a) life (b) stress against $b$ and $t$ for $\beta=0$.

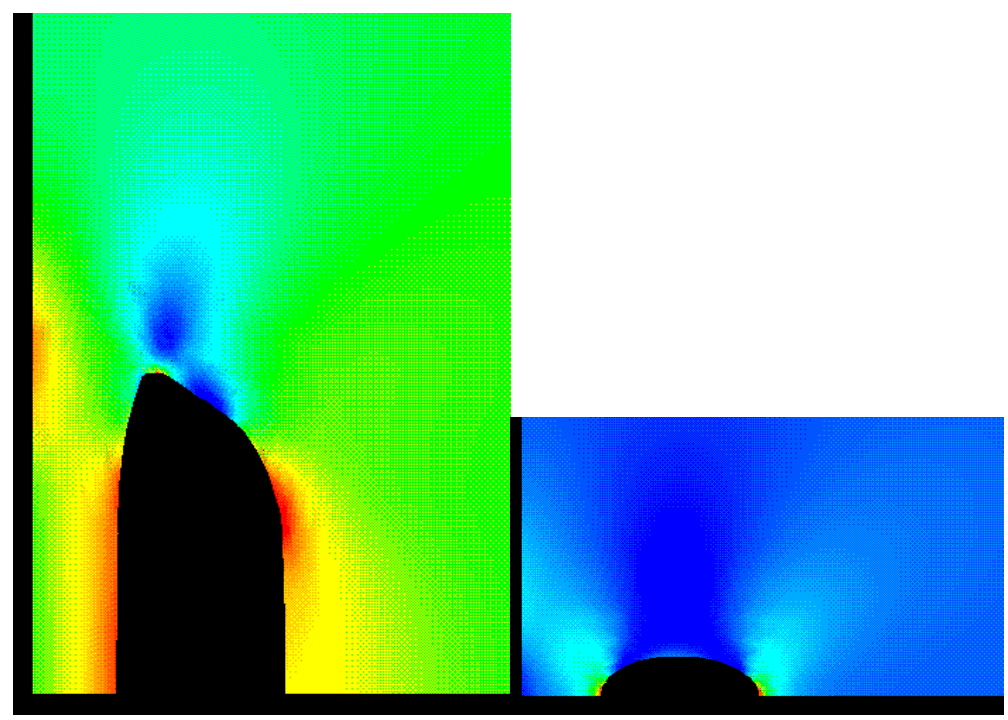

Fig. 4. Stress fields for hole shape (a) globally optimal (b) locally optimal. 